\title{
Titanium Rib Plate Technique for Huge Chest Wall Reconstruction
}

\author{
Yong Han Yoon \\ Department of Thoracic and Cardiovascular Surgery, Inha University College of Medicine, Incheon, Korea \\ E-mail: yoonkwon@inha.ac.kr \\ Received February 9, 2011; revised April 6, 2011; accepted August 2, 2011
}

\begin{abstract}
Chest wall reconstruction after en-bloc tumor resection is very important to preserve functional mobility and to enhance the cosmetic effect. Because they are flexible and pliable, titanium plates are proposed to reconstruct the chest wall, even though such chest wall reconstruction has been performed in only a few cases worldwide. We present a case of a 49-year-old man with a chondrosarcoma arising from the left $1^{\text {st }}$ rib, invading the manubrium, clavicle, $2^{\text {nd }}$ and $3^{\text {rd }}$ ribs, and the anterior segment of the left upper lobe. After wide resection, the chest wall was reconstructed using titanium rib plates and Marlex mesh - the Bovine pericardium sandwich type. The patient tolerated the pain well, and fourteen months after surgery, the chest wall was well preserved function mobility and improved pulmonary function test.
\end{abstract}

Keywords: Chest Wall Reconstruction, Titanium Plate, Chondrosarcoma

\section{Introduction}

Generally, chest wall defects larger than $5 \mathrm{~cm}$ in size, in any location, and those more than $10 \mathrm{~cm}$ in size, posterior or in close proximity to the scapula, must be reconstructed [1]. LeRoux and Shama [2], proposed that the ideal characteristics of a prosthetic material are rigidity to invalidate paradoxical chest wall motion, inertness to allow in-growth of fibrotic tissue and decrease the like hood to the appropriate shape at the time of operation, and radiolucency to allow radiographic follow-up the underlying problem. Techniques have been developed for thoracic reconstruction surgery that allows resection of chondrosarcomas. Wide excision surgical treatment is the best choice for chondrosarcoma, after which localized recurrence is the most common problem. Titanium plates have sufficient inertness to allow the in-growth of fibrous tissue [3]. Because rigid titanium plates have many advantages, we expect to use them widely for chest wall reconstruction.

\section{Method}

\section{Case Report}

A 48-year-old man was referred for evaluation of chest pain and a palpable chest wall mass. A chest computed tomography (CT) scan showed a large mass involving the left upper lobe, $1^{\text {st }}$ rib, and chest wall, originating from a well-defined $12.6 \times 10.1 \mathrm{~cm}$ chondrosarcoma in the anterior chest wall (Figures 1(a) and (b)). A localized chest wall biopsy showed a spindle cell sarcoma. The chest wall mass was resected. The wide excision was performed and included soft tissue, partial manubrium, clavicle, the $1^{\text {st }}-3^{\text {rd }}$ ribs, and anterior segment of the left upper lobe without any problems. The surgical margin had to be free of tumor by frozen section before the reconstructive procedure was attempted. Skeletal reconstruction surgery was performed, including the manubrium, clavicle, and $1^{\text {st }}-4^{\text {th }}$ ribs. We used a latissimus dorsi muscle flap associated with a split thickness skin graft. The pathology results revealed a dedifferentiated chondrosarcoma sarcoma, the 1st rib was a rhadomyosarcoma and cell necrosis extension into the intercostal soft tissue invaded the lung parenchyma and manubrium that was $16.0 \times 11.0 \times 11.0 \mathrm{~cm}$ in size. The patient was discharged uneventfully on post-operative day 24 and underwent radiotherapy.

\section{Surgical Technique}

Five rib plate surgery of the anterior chest wall was performed after resection of the chest wall chondrosarcoma, 


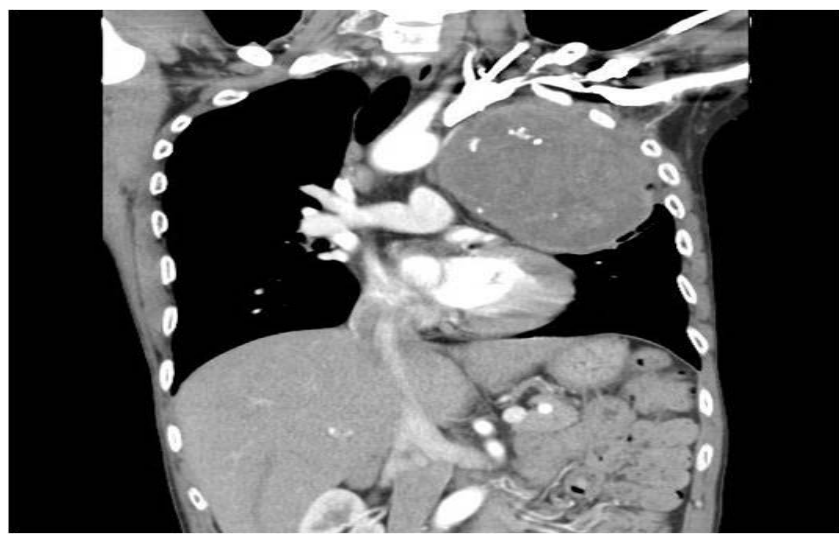

(a)

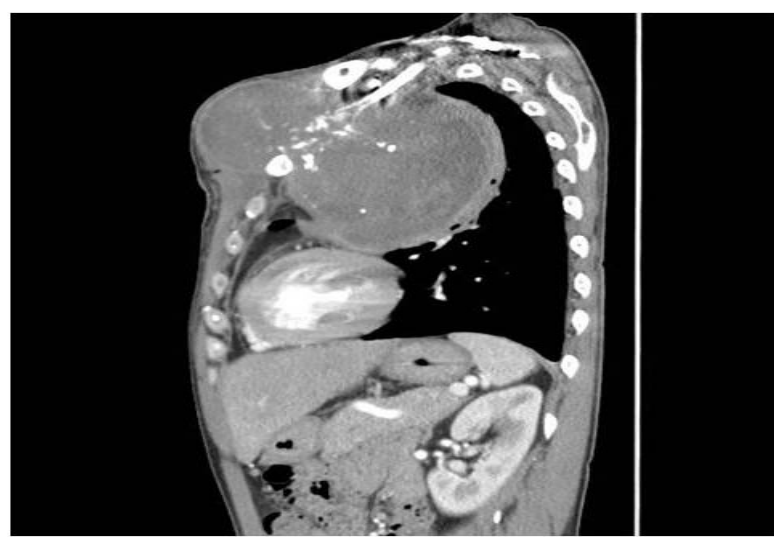

(b)

Figure 1. (a) Chest computed tomography scan showing the huge mass involving left upper lobe, $1^{\text {st }}$ rib and chest wall; (b) chest computed tomography scan showing the huge mass involving left anterior chest wall with sternum and left upper lobe.

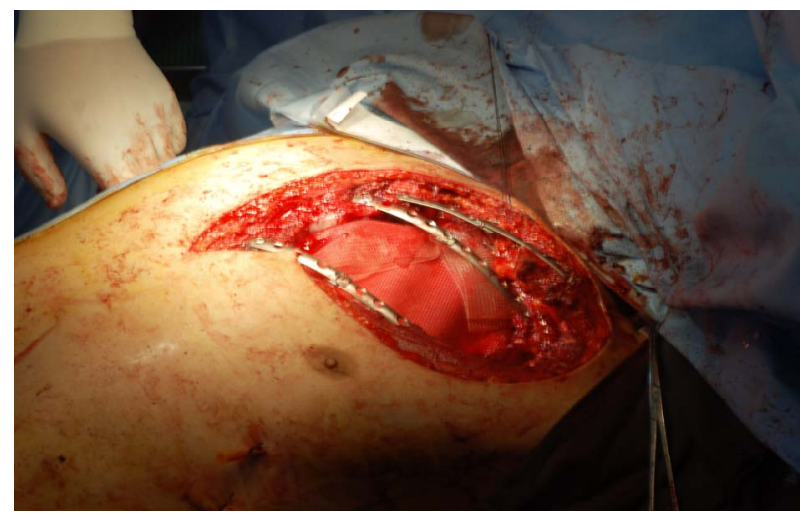

Figure 2. Intra-operative view of chest wall reconstruction, there was performed by double Marlex mesh-Bovine pericardium and multiple titanium rib plate.

partial clavicle, $1^{\text {st }}, 2^{\text {nd }}$, and $3^{\text {rd }}$ ribs, and the segmentectomy of the left upper lobe with severe adhesions between the chest walls mass and left upper lobe. We used a double Marlex mesh-Bovine pericardium composite to repair large defects involving the sternum and anterolateral chest wall that protect the underlying cardiovascular structures. The prosthesis was sewn with interrupted non-absorbable suture (2-0 Ethibond). Five rib plates were inserted between the $1^{\text {st }}, 2^{\text {nd }}, 3^{\text {rd }}$, and $4^{\text {th }}$ ribs, clavicle and manubrium (Figure 2). After the skeletal reconstruction was completed, the second phase of the chest wall reconstruction was performed with an interposition of large muscle and skin flaps.

\section{Postoperative Recovery}

The patient was discharged uneventfully on post-operative day 24 and underwent radiotherapy (Figure 3). The pulmonary function test results before and 4 months after surgery showed the following: vital capacity, 2.60 and 2.72 liter; $67 \%$ and 65\%; forced expiratory volume in 1

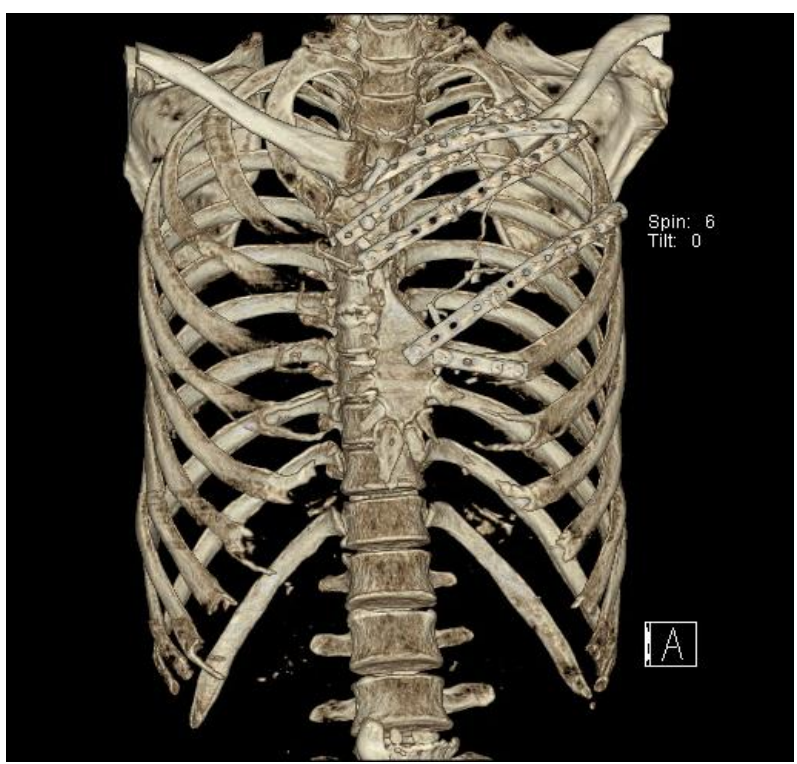

Figure 3. 3D chest wall computed tomography showing reconstructed left upper thoracic cage by multiple prosthesis and screw.

sec, 2.17 and 2.33 liter; $67 \%$ and $71 \%$. Fourteen months after surgery, the patient was doing well and had improved pulmonary function tests.

\section{Discussion}

Synthetic mesh (Prolene mesh, Gore-Tex, or Marelx mesh), methyl metacrylate, and a muscular flap are frequently used for chest wall reconstruction. Even though its safety has been studied and favorable results have been obtained [4-6], these meshes are too weak for lateral chest wall reconstruction [7]. Methyl methacrylate, popular in United States, can create good chest wall stability, but some problems have been reported in the 
preparation and adaptation to the shape of the defect [1]. Coonar et al. have reported on the advantages of titanium, including a high strength-to-weight ratio, osseointegration, and less interference with computed tomography than higher density metals have. A reduced rate of computed tomographic artifacts allows for more accurate three-dimensional reconstructions. Titanium is not ferromagnetic, so it can be used safely with magnetic resonance imaging [8]. In 1997, Nomori et al. performed chest wall reconstruction with a titanium hollow screw plate in a patient with squamous cell carcinoma invading the $2^{\text {th }}-4^{\text {th }}$ ribs [9]. The defected $3^{\text {rd }}$ and $4^{\text {th }}$ ribs were reconstructed with rib-to-rib titanium plates. At that time, meshes, such as PTFE patches, metal plates, and silastic sheeting were popular with reconstructing huge chest wall defects. We suspect those types of reconstructions presented very challenging trials, since there were no other published reports of titanium plate use in chest wall reconstruction for more than 10 years. In the past 2 years, a few excellent cases reports were published. Gonfiotti et al. operated on a Ewing's sarcoma patient: two STRATOS (Strasburg Thoracic Osteosyntheses System; MedXpert, Heitersheim, Germany) bars were fixed posteriorly to the ribs, with the special clips, and anterioly to the edges of the sternum, with metal wires [1]. Bille et al. operated on a Ewing's sarcoma patient with a Gore-tex mesh and titanium rib prosthesis to reconstruct the third, fourth and fifth ribs (Stratos) [7]. Coonar et al. placed four STRATOS titanium plates, fixed by clips to both sides of ribs. During the 21-month follow-up, there was no recurrence; also demonstrated were the durability and resistance of inserted titanium plates through scapular and elbow fractures [8,10]. Iarussi et al. performed 13 chest wall reconstructions using Synthes titanium plates (Synthes, West Chester, Pa) but no details were published [11]; even though they achieved chest cage stability, with only 1 titanium plate insertion in the resected area of the 2 nd rib. Just how this stability was achieved has not been discussed.

Compared to other reported cases, our patient had a very large mass; for that reason, extensive reconstruction from the clavicle to the $3^{\text {rd }}$ rib was performed. Instead of screws and wires, simpler and more relevant material, such as clips, likely make it easier to fix plates. We used titanium plates for the first time for a large chest wall construction, and we had no difficulty inserting the plates and we easily understood how they work. We assume that Titanium plates cause less severe adhesions than other rigid and non-rigid prostheses. Titanium plate chest wall reconstruction is not used worldwide but has been performed in some European countries. To establish the most effective and optimal procedure, long-term follow up and multicenter studies will be necessary. Titanium rib plate is suitable in chest wall reconstruction surgery for large defect chest walls and facilitates a good quality of life.

Comparisons between titanium plates and other methods for cost-effectiveness, quality of life, convenience of skill, recurrence rate, and mortality should be analyzed in near future.

\section{Conclusions}

Titanium rib plate is suitable in chest wall reconstruction surgery for huge defect chest walls and facilitates a good quality of life.

\section{Acknowledgements}

This work was supported by an Inha University Research Gant.

\section{References}

[1] A. Gonfiotti, P. F. Santini, D. Campanacci, et al., "Malignant Primary Chest-Wall Tumors: Techniques of Reconstruction and Survival,” European Journal of CardioThoracic Surgery, Vol. 38, No. 1, 2010, pp. 39-45. doi:10.1016/j.ejcts.2009.12.046

[2] B. R. LeRoux and D. M. Shama, "Resection of Tumors of the Chest Wall," Current Problems in Surgery, Vol. 20, No. 6, 1983, pp. 345-386.

doi:10.1016/S0011-3840(83)80007-0

[3] H. Horio, T. Ohtshka, Y. Kubota, T. Hasegwa, H. Nomori, T. Naruke and K. Suemasu, "Large Chest Wall Resconstruction Using Titanium Micromesh and Pedicled Lastissimus Dorsi Muscularcutaneous Flap: Report of a Case,” Surgery Today, Vol. 35, No. 1, 2005, pp. 73-75. doi:10.1007/s00595-004-2868-4

[4] N.C. Dang, S. Siegel and J. D. Philips, "Malignant Chest Wall Tumors in Children and Young Adults," Journal of Pediatric Surgery, Vol. 34, No. 12, 1999, pp. 1773-1778. doi:10.1016/S0022-3468(99)90310-X

[5] Mansour K. A., Thourani V. H., Losken A., Reeves J. G., Miller J. I. and Jones G. E., Chest wall resection and reconstruction: a 25-year experience. The Annals of Thoracic Surgery, Vol. 73, No. 6, 2002, pp. 1720-1725. doi:10.1016/S0003-4975(02)03527-0

[6] P. G. Arnold and P. C. Pairolero, "Chest-Wall Reconstruction: An Account of 500 Consecutive Patients," Plastic and Reconstructive Surgery, Vol. 98, No. 5, 1996, pp. 804-810. doi:10.1097/00006534-199610000-00008

[7] A. Bille, M. Gisabella, L. Errico and P. Borasio, “A Suitable System of Reconstruction with Titanium Rib Prosthesis after Chest Wall Resection for Ewing Sarcoma,” Interactive CardioVascular and Thoracic Surgery, Vol. 12, No. 2, 2011, pp. 293-296.

doi:10.1510/icvts.2010.245902 
[8] A. S. Coonar, N. Qureshi, I. Smith, F. C. Wells and E. Reisberg and J.-M. Wihlm, “A Novel Titanium Rib Bridge System for Chest Wall Reconstruction,” The Annals of Thoracic Surgery, Vol. 87, No. 5, 2009, pp. e46-e48. doi:10.1016/j.athoracsur.2009.01.069

[9] H. Nomori, H. Horio and T. Hasegawa, "Chest Wall Reconstruction Using Titanium Hollow Screw Reconstruction Plate," The Journal of Thoracic and Cardiovascular Surgery, Vol. 45, No. 1, 1997, pp. 35-37. doi:10.1055/s-2007-1013681

[10] A. S. Coonar, J. M. Wihlm, F. C. Wells and N. Qureshi, "Intermediate Outcome and Dynamic Computerized To- mography after Chest Wall Reconstruction with the STRTOS Titanium Rib Bridge System: Videodemonstration of Preserved Bucket-Handle Rib Motion,” Interactive CardioVascular and Thoracic Surgery, Vol. 12, 2011, pp. 80-81. doi:10.1510/icvts.2010.249615

[11] T. Iarussi, A. Pardolesi, P. Camplese and R. Sacco, "Composite Chest Wall Reconstruction Using Titanium Plates and Mesh Preserves Chest Wall Function," The Journal of Thoracic and Cardiovascular Surgery, Vol. 140, No. 2, 2010, pp. 476-7. doi:10.1016/j.jtcvs.2009.07.030 